\title{
Transhepatic Approach to Create Stent Fenestration in the Extracardiac Fontan Conduit in a Child With Dextrocardia and Interrupted Inferior Vena Cava With Azygos Continuation
}

\author{
Daisuke Kobayashi, ${ }^{\star}$ MD, Daniel R. Turner, MD, and Thomas J. Forbes, MD \\ Plastic bronchitis is a rare life-threatening complication of Fontan operation. When \\ medical treatment is ineffective in the setting of high systemic venous pressures, Fon- \\ tan fenestration may be considered to decompress venous pressures and improve car- \\ diac output by creation of the right-to-left shunting. However, transcatheter approach \\ can be difficult in patients with complex venous anatomy. We report a 4-year-old girl \\ born with hypoplastic left ventricle and heterotaxy syndrome, who developed plastic \\ bronchitis following extracardiac Fontan procedure. Her venous anatomy was complex \\ with dextrocardia and interrupted inferior vena cava with azygos continuation. Stent \\ fenestration was successfully performed via transhepatic approach, which was \\ selected based on the anatomical relationship (between extracardiac conduit, left \\ atrium, and hepatic veins) delineated by pre-catheterization cardiac MRI. Simultaneous \\ transesophageal echocardiography guided the intervention. Her plastic bronchitis \\ improved significantly in 3 months but slowly progressed after the stent fenestration. \\ At her 8-month follow-up, stent fenestration remains open and she is currently under \\ heart transplantation evaluation due to persistent plastic bronchitis. Treatment of plas- \\ tic bronchitis can be undertaken with Fontan fenestration, with pre-procedural \\ MRI playing an essential role in patients with complex venous anatomy. ( 2012 Wiley \\ Periodicals, Inc.
}

Key words: extracardiac conduit; fenestration; plastic bronchitis; Fontan operation; heterotaxy

\section{INTRODUCTION}

Plastic bronchitis is a rare but severe complication in patients following Fontan palliation [1]. Surgical or transcatheter creation/dilation of fenestration of the Fontan atrial baffle may be considered as plastic bronchitis can be life-threatening and is often refractory to medical treatment $[2,3]$. Transcatheter creation of Fon$\tan$ fenestration has been reported but can be technically difficult in cases of complex venous anatomy [4]. We report a 4-year-old girl with hypoplastic left heart and heterotaxy syndrome, who developed plastic bronchitis following extracardiac Fontan procedure. Her venous anatomy was complex with dextrocardia, interrupted inferior vena cava (IVC) with azygos connection, and a midline liver. The stent fenestration was successfully performed of the extracardiac conduit via transhepatic approach.
Division of Pediatric Cardiology, Children's Hospital of Michigan, Carman and Ann Adams Department of Pediatrics, Wayne State University School of Medicine, Detroit, Michigan

Conflict of interest: Nothing to report.

*Correspondence to: Daisuke Kobayashi, MD, Division of Cardiology, Carman and Ann Adams Department of Pediatrics, Children's Hospital of Michigan, Wayne State University School of Medicine, 3901 Beaubien Blvd, Detroit, MI 48201-2119.

E-mail: dkobayas@dmc.org

Received 15 December 2011; Revision accepted 30 June 2012

DOI 10.1002/ccd.24551

Published online 7 August 2012 in Wiley Online Library (wileyonlinelibrary.com) 


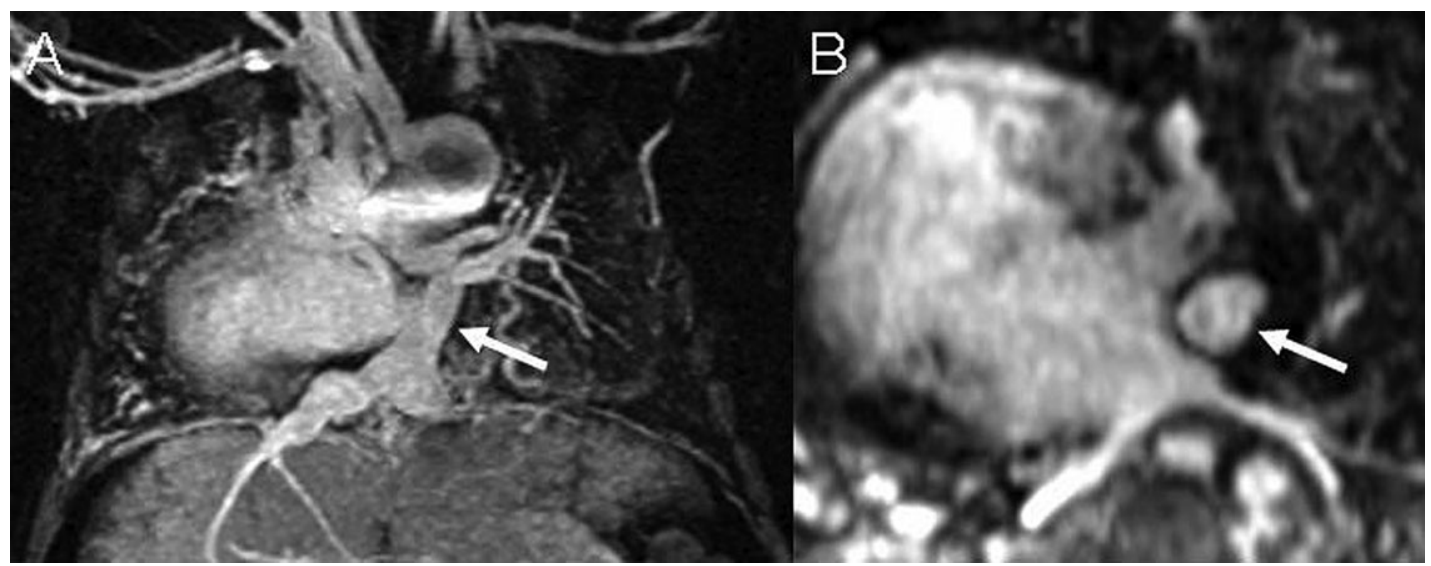

Fig. 1. Cardiac MRI. A: Coronal view, showing hepatic vein joining to the extracardic Fontan conduit (arrow). B: Axial view, showing the proximity between the conduit (arrow) and atrial wall with the distance measuring $2.8 \mathrm{~mm}$.

\section{CASE REPORT}

A 4-year-old $14.5 \mathrm{~kg}$ girl with history of extracardiac Fontan procedure for hypoplastic left heart and heterotaxy syndrome was brought to the cardiac catheterization laboratory for transcatheter fenestration creation of her Fontan conduit. Her cardiac anatomy included dextrocardia, situs ambigious, aortic and mitral valve atresia, left aortic arch, hypoplastic left ventricle and ascending aorta, common atrium, and interrupted IVC with azygos continuation to the right-sided superior vena cava (SVC). She underwent initial Hybrid procedure consisting of bilateral pulmonary artery banding and stenting of her patent ductus arteriosus at 5 weeks of age. She underwent bidirectional cavo-pulmonary anastomosis with Damus-Kaye-Stansel procedure and aortic arch repair at 7 months of age. Eventually, she underwent modified Fontan procedure with an extracardiac tunnel at 3 years of age. The hepatic veins were anastomosed with a $14 \mathrm{~mm}$ Gore-Tex graft to create the extracardiac tunnel. The postoperative course following Fontan palliation was complicated with persistent chylous pleural effusion requiring mechanical pleurodesis, thoracic duct ligation, and surgical fenestration of the Fontan conduit. Her pleural and pericardial effusions slowly resolved with vigorous diuretic therapy. However, over the following 6 months she developed plastic bronchitis requiring frequent hospitalizations. Her plastic bronchitis was refractory to the medical therapy including sildenafil, steroid, sodium chloride inhalation, and chest physiotherapy. Her surgically created fenestration closed spontaneously. The single ventricle function was normal with trivial atrioventricular valve insufficiency. Her systemic saturations were in the low to mid 70s in room air. Her chest X-ray showed bilateral persistent patchy opac- ities, most prominent in the left lung base. The decision was made to create a Fontan fenestration to decompress the systemic venous pressures and improve cardiac output via the transhepatic route. Prior to the catheterization, cardiac MRI was obtained to delineate her complex venous anatomy and the anatomical relationship of the extracardiac atrial baffle with her left atrium (Fig .1).

Cardiac catheterization was performed with the patient being mechanically ventilated under general anesthesia. Using a 5-Fr sheath, a 4-Fr JB Glide catheter was advanced using a $0.035^{\prime \prime}$ glide wire from the right femoral vein to azygos vein, SVC, extracardiac conduit, and hepatic veins. This assisted us in determining which hepatic vein had the most direct course to the extracardiac conduit, which we used to guide our transhepatic needle access. The mean pressure of the Fontan conduit was $22 \mathrm{~mm} \mathrm{Hg}$. Hand injection was performed in the extracardiac conduit. The fenestration was completely closed with significant blood stasis being observed within the conduit. There was no stenosis or obstruction in the pulmonary arterial or atrial baffle system. Using the transhepatic approach, the wire was successfully advanced from the middle hepatic vein to Fontan conduit and a 6-Fr sheath was placed. The sheath was exchanged over a $0.035^{\prime \prime}$ Rosen wire for a 6-Fr Mullins sheath, which was positioned in the inferior portion of conduit. Under transesophageal echocardiographic guidance, transeptal puncture was successfully performed to create the fenestration between the extracardiac conduit and the atrium (Fig. 2). A 19G transeptal needle was advanced postero-medially from the Fontan conduit toward the atrium (Fig. 3). The sheath was further advanced into the atrium, where a hand injection was performed to confirm the position. The mean pressure of the atrium was $11 \mathrm{~mm} \mathrm{Hg}$. 


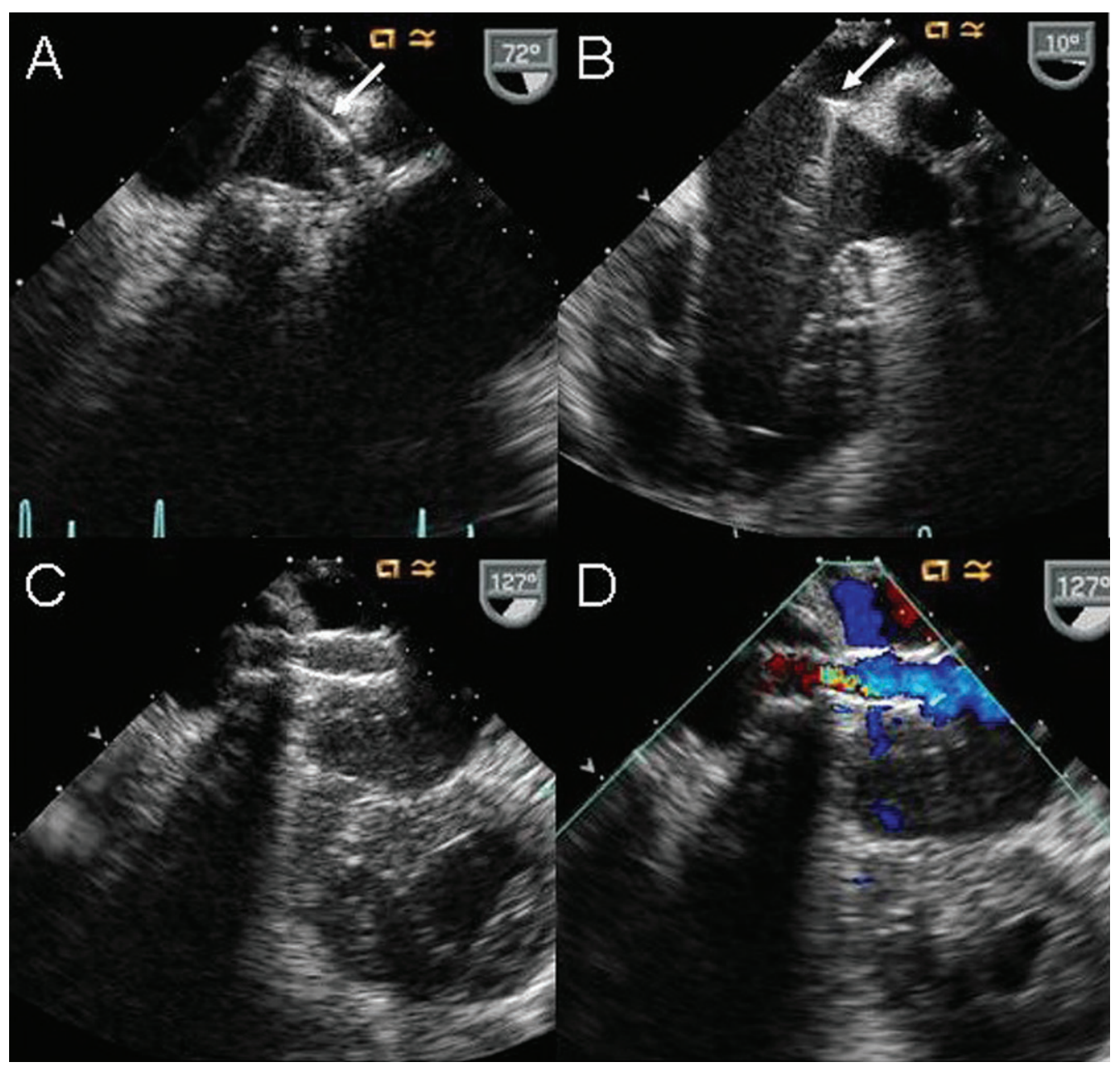

Fig. 2. Transesophageal echocardiography. A: Transseptal needle (arrow) is directed from the conduit to common atrium. B: Transseptal needle (arrow) is inserted into the atrium. C: The stent is positioned across the tissue between the conduit and atrium. D: Doppler interrogation shows the blood shunting from the conduit to the atrium. [Color figure can be viewed in the online issue, which is available at wileyonlinelibrary.com.]

Without pre-dilation, the stent fenestration was performed. A Palmaz Blue 2407 stent premounted $7 \mathrm{~mm}$ $\times 24 \mathrm{~mm}$ balloon catheter was positioned across the tissue between the conduit and atrium over a $0.018^{\prime \prime}$ Flex-T wire. The stent was deployed with a gradual inflation of balloon to a maximum of 5.6 atmospheres, leaving a "dog bone shape" to prevent stent dislodgement. The narrowest portion of stent measured 5.4 $\mathrm{mm}$. Transesophageal echocardiogram confirmed stable stent position with right to left shunt flow across the stent. Post-stent, the mean pressure of conduit and atrium were 19 and $11 \mathrm{~mm} \mathrm{Hg}$, respectively. Angiography in the conduit showed stable position of stent (Fig. 3). Hemostasis was achieved by the application of external pressure on the liver for $20 \mathrm{~min}$.

Post-catheterization course was uncomplicated. No intraperitoneal hemorrhage was noted on abdominal ultrasound. Clopidogrel was started in addition to aspi- rin to maintain stent patency. That same evening the patient's saturations increased to the low to mid $80 \mathrm{~s}$ in room air. She was discharged home the following morning. At 3-month follow-up, there was no recurrence of plastic bronchitis. Her oxygen saturations increased to $85 \%$ on room air. However, over the following 6 months her plastic bronchitis slowly progressed with worsening ascites. Heart transplantation evaluation is currently being pursued. Chest CT (7 months after stent fenestration) showed stable position of stent fenestration in the wall of extracardiac conduit and persistent left lower lobe atelectasis (Fig. 4A and B). As a part of heart transplantation evaluation, cardiac catheterization was performed to obtain hemodynamic information. The mean systemic venous pressure was $18 \mathrm{~mm} \mathrm{Hg}$. The right and left mean pulmonary artery capillary wedge pressures were 13 and $21 \mathrm{~mm} \mathrm{Hg}$, respectively. Angiography in the extracardiac conduit 


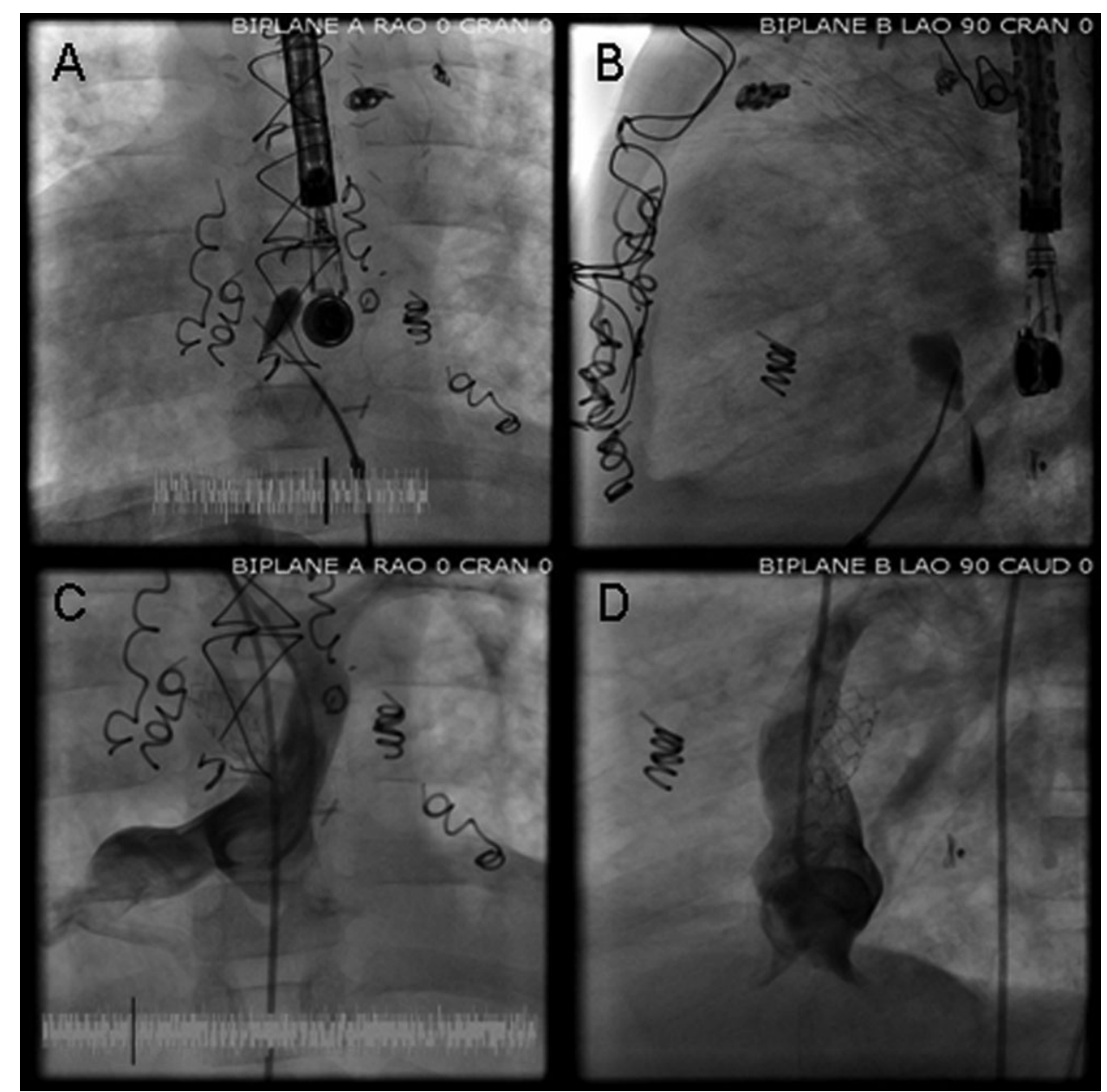

Fig. 3. Transseptal puncture in (A) antero-posterior and (B) lateral view. Contrast staining is seen in the tissue between extracardiac Fontan conduit and atrial wall. Angiography in the extracardiac Fontan conduit after stent placement in the created fenestration in (C) anteroposterior and (D) lateral view.

showed right-to-left shunting across the fenestration. Left pulmonary artery (LPA) was not filled by angiography in neither SVC nor Fontan conduit (Fig. 4C). Further imaging revealed a large collateral vessel originating from the innominate vein draining into the left pulmonary vein (Fig. 4D). The collateral vessel was occluded with a $0.038^{\prime \prime}$ Gianturco coil. At latest follow-up (8 months), stent fenestration remains open with improved flow into the LPA being observed.

\section{DISCUSSION}

Plastic bronchitis is a rare and potentially life-threatening disease in which bronchial casts are formed within the airways that occlude the bronchus [5]. Plastic bronchitis is one of the challenging complications in patients with Fontan physiology [1]. The casts following the Fontan operation are often described as acellular and composed of mucin [1]. The etiology of plastic bronchitis following Fontan palliation remains unknown. One theory states that dysfunction of the bronchial membrane with subsequent break in the integrity of the bronchial mucosa due to Fontan circulation leads to leakage of proteinaceous material into the airway [5]. The management of plastic bronchitis is difficult. Standard therapies include inhaled steroids, albuterol, pulmonary physiotherapy, acetylcysteine, and bronchoscopic removal of casts [5]. The use of aerosolized tissue plasminogen activator has been reported [6]. Specific treatments in patients following Fontan palliation include the correction of any right sided obstructions and optimizing cardiac rhythm and cardiac output [1]. Fenestration of the Fontan circuit is another therapeutic option used to decrease venous pressures and improve cardiac output $[2,3]$. Our patient had frequent episodes of plastic bronchitis flare in spite of aggressive medical therapy. Her desaturation was caused by ventilation-perfusion mismatch due to 


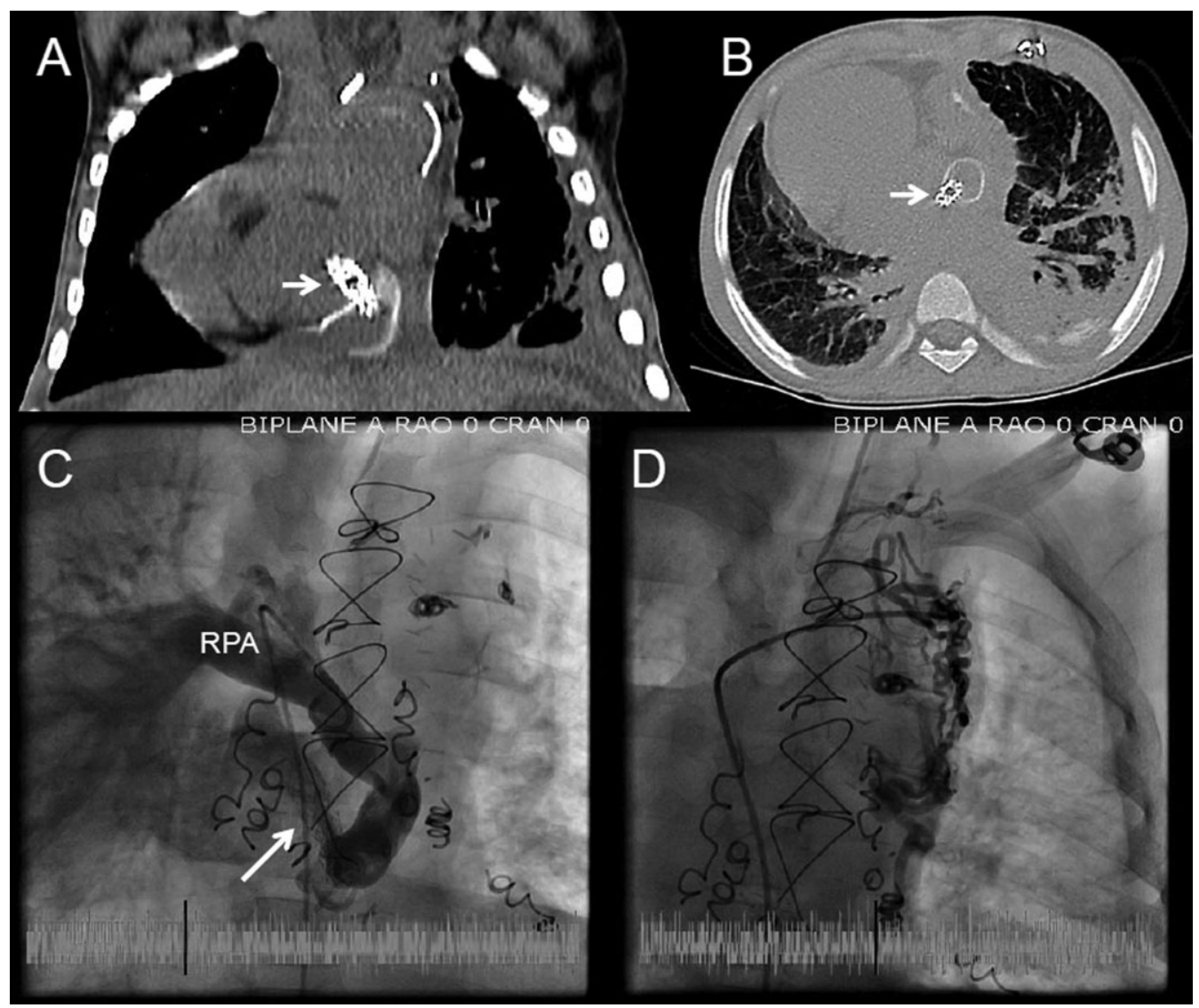

Fig. 4. CT of chest in the coronal (A) and axial view (B). The stent (arrow) remains in a stable position. The atelectasis is prominent in the left lung base. (C) Angiography in the Fontan conduit showed the shunting blood through the stent fenestration (arrow). The blood exclusively flows into the right pulmonary artery (RPA). The pigtail catheter is advanced from right femoral vein, to the azygous vein, SVC and Fontan conduit. (D) The selective angiography in the origin of a large collateral vein, which originates from the left innominate vein. The collateral veins eventually drain into the left pulmonary vein. The catheter is advanced from right femoral vein to azygos vein, SVC and left innominate vein. persistent atelectasis associated with plastic bronchitis in addition to a veno-venous collateral. The systemic venous pressure was elevated at mean of $22 \mathrm{~mm} \mathrm{Hg}$. We were surprised at the immediate clinical improvement observed following creation of the fenestration in the Fontan circuit in our patient. The patient's oxygen saturation increased from $72 \%$ to $85 \%$ prior to leaving the catheterization lab in spite of right-to-left shunting at the atrial baffle level. Her pulmonary symptomatology completely resolved the following morning, with her continuing to do well 3 months after the procedure. Improvement of perfusion-ventilation mismatches undoubtedly contributed to her improved overall arterial oxygenation.

Surgically created fenestration of the Fontan conduit has shown to reduce venous pressures and increase cardiac output, leading to lower postoperative morbidity and mortality [7]. Kreutzer et al. reported the transcatheter baffle fenestration creation and/or dilation in 14 symptomatic Fontan patients [4]. Complex venous anatomy is a particular challenge to perform the transcatheter fenestration creation. The femoral vein access may not provide a stable needle position to perforate the smooth surface of Gore-Tex material in the extracardiac conduit [8]. Transhepatic approach can stabilize needle position, allowing a more perpendicular approach to the conduit [8]. Kenny et al. reported a 3-year-old boy with hypoplastic left heart syndrome, who underwent transcatheter stent fenestration in extracardiac Fontan conduit [8]. This patient had levocardia without interrupted IVC. To create a diabolo-shape configuration of stent, a $4 \mathrm{~mm} 2-0$ Prolene suture was tied around the mid-portion of the Palmaz Genesis PG1910 bare metal stent (Cordis J\&J, Warren, NJ) mounted on $12 \mathrm{~mm}$ Opta balloon catheter (Cordis J\&J, Warren, NJ). This suture technique facilitated the stent flaring in the both ends with a central waist. In contrast, our patient had more complex venous anatomy including dextrocardia and interrupted IVC, precluding us from using the usual femoral access. Furthermore, 
right SVC combined with the extracardiac conduit made it difficult for us to come from the right internal jugular vein with the transeptal needle. Though there have been instances where perforating across the inferior part of the pulmonary artery into the superior portion of the left atrium has been done to create an atrial baffle fenestration [9], we decided to choose the transheptatic route for our patient. Prior to the catheterization, cardiac MRI (Fig. 1) indicated that using the midline liver, along the left vertebral line, would be the most direct route to cross the extracardiac conduit. Access to the correct hepatic vein was assisted by entering into the right femoral vein. A JB glide catheter (Cook corp, Bloomington, IN) and 0.035" glide wire (Terumo corp, Tokyo, Japan) was used to traverse the azygous continuation of the IVC, through the SVC-pulmonary anastomosis, enter into the extracardiac atrial baffle, and advance into the correct heptatic vein. This served as a marker for us to obtain access to the most direct hepatic connection to the atrial baffle.

The technique for creation of a de novo fenestration of an extracardiac Gore-Tex conduit has been previously described by Bae et al. [10]. Perforating the fenestration in the extracardic conduit can be safely performed after a few months, when the scar tissue likely fills the space between the conduit and the atrial wall and prevent bleeding [10]. Though it has been advocated by some to use a covered stent when creating an extracardiac baffle fenestration [9], we decided to use the pre-mounted $7 \mathrm{~mm} \times 24 \mathrm{~mm}$ length bare metal Palmaz Blue (Cordis corp, Warren, NJ). The flaring both ends of stent was achieved without the suture technique (mentioned above) to prevent late stent migration. The balloon was gradually inflated to create the dog-bone shape configuration of stent. Finally, when difficulties have been encountered in crossing the atrial baffle due to the inability to achieve perpendicular orientation of the transeptal needle with the atrial baffle, the author's have found it helpful to change the patient's position on the table (for example, in a patient with levocardia, flexing the right side of the hip), to reposition the atrial septum to be more perpendicular to the needle.

\section{CONCLUSION}

We report successful stent fenestration of an extracardiac Fontan conduit in a patient with complex venous anatomy suffering from plastic bronchitis. Due to the complex nature of this patient's anatomy, performance of a cardiac MRI was essential for both the planning and successful completion of this procedure.

\section{AUTHOR CONTRIBUTION}

DK is a first author who wrote the manuscript. DRT is a pediatric interventional cardiologist and critically edited the manuscript from the interventional standpoint. TJF is the senior author and critically edited and approved the manuscript.

\section{REFERENCES}

1. Madsen P, Shah SA, Rubin BK. Plastic bronchitis: New insights and a classification scheme. Paediatr Respir Rev 2005;6:292-300.

2. Wilson J, Russell J, Williams W, Benson L. Fenestration of the Fontan circuit as treatment for plastic bronchitis. Pediatr Cardiol 2005;26:717-719.

3. Chaudhari M, Stumper O. Plastic bronchitis after Fontan operation: Treatment with stent fenestration of the Fontan circuit. Heart 2004;90:801.

4. Kreutzer J, Lock JE, Jonas RA, Keane JF. Transcatheter fenestration dilation and/or creation in postoperative Fontan patients. Am J Cardiol 1997;79:228-232.

5. Goldberg DJ, Dodds K, Rychik J. Rare problems associated with the Fontan circulation. Cardiol Young 2010;20:113-119.

6. Costello JM, Steinhorn D, McColley S, Gerber ME, Kumar SP. Treatment of plastic bronchitis in a Fontan patient with tissue plasminogen activator: A case report and review of the literature. Pediatrics 2002;109:e67.

7. Bridges ND, Mayer JE Jr, Lock JE, Jonas RA, Hanley FL, Keane JF, Perry SB, Castaneda AR. Effect of baffle fenestration on outcome of the modified Fontan operation. Circulation 1992;86:1762-1769.

8. Kenny D, McMahon C, Walsh KP. Transhepatic approach for extracardiac inferior cavopulmonary connection stent fenestration. Congenit Heart Dis 2011;6:276-279.

9. Mehta C, Jones T, De Giovanni JV. Percutaneous transcatheter communication between the pulmonary artery and atrium following an extra-cardiac Fontan: An alternative approach to fenestration avoiding conduit perforation. Catheter Cardiovasc Interv 2008;71:936-939.

10. Bae EJ, Choi JY, Noh CI, Yun YS, Lee JR. De novo creation of fenestration and stent implantation for failed extracardiac conduit Fontan operation. Int J Cardiol 2003;88:321-322. 